\title{
Stability and Passivity Analysis for Lur'e Singular Systems with Markovian Switching
}

\author{
Ping-Fang Zhou Yue-Ying Wang Quan-Bao Wang Ji-An Chen Deng-Ping Duan \\ School of Aeronautics and Astronautics, Shanghai Jiao Tong University, Shanghai 200240, China
}

\begin{abstract}
This paper is concerned with the stochastic stability and passivity analysis for a class of Lur'e singular systems with time-varying delay and Markovian switching. By using the free-weighting matrices approach, a delay-dependent stability criterion, which guarantees that the system is stochastically stable and robustly passive, is derived in terms of linear matrix inequality (LMI). Two numerical examples are provided to illustrate the effectiveness of the proposed method.
\end{abstract}

Keywords: Lur'e singular time-delay systems, Markovian jump systems, delay-dependent, passivity, linear matrix inequality (LMI).

\section{Introduction}

The problem of stability analysis for Lur'e control systems has been a topic of recurring interest over the past decades due to the fact that a large class of nonlinear systems in engineering can be modeled as this class of systems. A great number of results related to such systems have been reported in the literatures [1-3]. Recently, much attention has been paid to the study of Lur'e singular systems. The control problem for singular systems of such class is more complicated than for regular ones, because the singular systems usually have three types of mode, namely, finite-dynamic mode, impulse mode and non-dynamic mode, while the latter two do not appear in state space. The results related to Lur'e singular systems can be classified into two types: delay-independent cases $^{[4,5]}$ and delay-dependent cases $^{[6-10]}$. It should be pointed out that the delay-dependent results are less conservative than the delay-independent ones, especially when the time delays are small. Till now, some results have been reported related to singular/regular systems with Markovian switching ${ }^{[11-15]}$. However, to the best of our knowledge, the delay-dependent stability analysis for Lur'e singular systems with Markovian switching has not been investigated.

On the other hand, the notion of passivity has played an important role in analysis and control design of linear and nonlinear systems. Passivity is part of a broader and more general theory of dissipativity, and it can maintain the internal stability of the system ${ }^{[16,17]}$. So it is necessary to investigate the passivity of systems. However, the passivity analysis for Lur'e systems has been paid little attention.

Motivated by the aforementioned factors, the problem of delay-dependent stability and passivity for Lur'e singular systems with Markovian switching is considered in this paper. The free-weighting matrices approach is employed to derive the delay-dependent criterion, which guarantees that the system is regular, impulse free, stochastically stable, and robustly passive. Two numerical examples are provided to illustrate the effectiveness of the proposed method.

Manuscript received February 24, 2012; revised August 3, 2012

This work was supported by National High Technology Research and Development Program of China (863 Program) (No. 2011AA7052011).

\section{Problem formulation}

Consider a class of Lur'e singular systems with Markovian switching and time-varying delay described as

$$
\left\{\begin{aligned}
E \dot{x}(t)= & A\left(r_{t}\right) x(t)+A_{d}\left(r_{t}\right) x(t-d(t))+ \\
& D\left(r_{t}\right) f\left(\sigma\left(r_{t}\right), r_{t}\right)+B\left(r_{t}\right) w(t) \\
z(t)= & C_{z}\left(r_{t}\right) x(t)+C_{d z}\left(r_{t}\right) x(t-d(t))+B_{z}\left(r_{t}\right) w(t) \\
\sigma\left(r_{t}\right)= & C\left(r_{t}\right) x(t) \\
x(t)= & \phi(t), \forall t \in[-\bar{d}, 0]
\end{aligned}\right.
$$

where $x(t) \in \mathbf{R}^{n}$ is the state vector, $z(t) \in \mathbf{R}^{s}$ is the controlled output, $w(t) \in \mathbf{R}^{p}$ is the disturbance input which belongs to $\mathbf{L}_{2}[0, \infty), E \in \mathbf{R}^{n \times n}$ may be singular, which is assumed that $\operatorname{rank}(E)=r \leqslant n, \phi(t) \in \mathbf{C}_{n, \bar{d}}$ is a compatible vector valued initial function, $A\left(r_{t}\right), A_{d}\left(r_{t}\right), B\left(r_{t}\right)$, $D\left(r_{t}\right), C_{z}\left(r_{t}\right), C_{d z}\left(r_{t}\right), B\left(r_{t}\right), B_{z}\left(r_{t}\right)$ and $C\left(r_{t}\right)$ are real constant matrices of appropriate dimensions, and $\left\{r_{t}\right\}$ is a continuous-time Markov process with a right continuous trajectory taking values finite set $\mathbf{S}=\{1,2, \cdots, N\}$ with transition probabilities as

$$
\boldsymbol{P}\left[r_{t+\Delta t}=j \mid r_{t}=i\right]=\left\{\begin{array}{l}
\pi_{i j} \Delta+o(\Delta), \text { if } j \neq i \\
1+\pi_{i j} \Delta+o(\Delta), \text { if } j=i
\end{array}\right.
$$

where $\lim _{\Delta \rightarrow 0} \frac{o(\Delta)}{\Delta}=0, \pi_{i j}>0, j \neq i$ and $\pi_{i i}=$ $-\sum_{j \neq i} \pi_{i j}$ for each $i \in \mathbf{S}, \sigma(r t)=\left(\sigma_{r t 1}, \sigma_{r t 2}, \cdots, \sigma_{r t q}\right)^{\mathrm{T}}$, $f_{r t}\left(\sigma_{i}\right)=\left(f_{r t 1}\left(\sigma_{i 1}\right), f_{r t 2}\left(\sigma_{i 2}\right), \cdots, f_{r t q}\left(\sigma_{i q}\right)\right)^{\mathrm{T}}$. The time delay $d(t)$ is a time-varying continuous function that satisfies

$$
0 \leqslant d(t) \leqslant \bar{d}, \quad 0 \leqslant \dot{d}(t) \leqslant \mu \leqslant 1, \quad \forall i \in \mathbf{S}
$$

where $\bar{d}$ and $\mu$ are constants.

For notational simplicity, in the sequel, for each possible $r_{t} \in i, i \in \mathbf{S}$, a matrix $M\left(r_{t}\right)$ will be denoted by $M_{i}$. For example, $A\left(r_{t}\right)$ is denoted by $A_{i}, A_{d}\left(r_{t}\right)$ is denoted by $A_{d i}$, etc.

The nonlinear feedback path is formulated as

$$
\begin{aligned}
& f_{i}\left(\sigma_{i}\right)=\left(f_{i 1}\left(\sigma_{i 1}\right), f_{i 2}\left(\sigma_{i 2}\right), \cdots, f_{i m}\left(\sigma_{i m}\right)\right)^{\mathrm{T}} \\
& \sigma_{i}=\left(\sigma_{i 1}, \sigma_{i 2}, \cdots, \sigma_{i m}\right)^{\mathrm{T}}
\end{aligned}
$$


with each $f_{i j}(\cdot)$ satisfying the finite sector condition:

$$
\begin{aligned}
& f_{i j}(\cdot) \in K_{j}\left[0, k_{j}\right]= \\
& \quad\left\{f_{i j}(\cdot) \mid f_{i j}(0)=0,0<\sigma_{i j} f_{i j}\left(\sigma_{i j}\right) \leqslant k_{j} \sigma_{i j}^{2}, \sigma_{i j} \neq 0\right\} \\
& \quad j=1,2, \cdots, m, \forall i \in \mathbf{S} .
\end{aligned}
$$

The nominal unforced system of (1) can be written as

$$
E \dot{x}(t)=A_{i} x(t)+A_{d i} x(t-d(t)) .
$$

Throughout this paper, we shall use the following concepts and introduce the following useful lemmas.

\section{Definition 1.}

1) System (4) is said to be regular and impulse free for any time delay $d(t)$ satisfying $(2)$, if the pairs $\left(E, A_{i}\right)$ and $\left(E, A_{i}+A_{d i}\right)$ are regular and impulse free for every $i \in \mathbf{S}$.

2) System (4) is said to be stochastically stable for any $f_{i j}(\cdot)(j=1,2, \cdots, q)$ satisfying $(3)$, if there exists a constant $T\left(r_{0}, \phi(\cdot)\right)$ such that

$$
\mathrm{E}\left[\int_{0}^{\infty}\|x(t)\|^{2} \mathrm{~d} t \mid r_{0}, x(s)=\phi(s), s \in[-\bar{d}, 0]\right] \leqslant T\left(r_{0}, \phi(\cdot)\right) .
$$

3) System (4) is said to be stochastically admissible, if it is regular, impulse free and stochastically stable.

Definition 2. The Lur'e singular system (1) is said to be robustly passive, if there exists a scalar $\gamma \geqslant 0$ such that

$$
\mathrm{E}\left\{2 \int_{0}^{t^{*}} w^{\mathrm{T}}(s) z(s) \mathrm{d} s\right\} \geqslant-\gamma \int_{0}^{t^{*}} w^{\mathrm{T}}(s) w(s) \mathrm{d} s
$$

for all $t^{*}>0$ under zero initial conditions, $w(t) \in \mathbf{L}_{2}[0, \infty)$.

Lemma 1 (S-procedure) ${ }^{[\mathbf{1 8}]}$. Let $F_{i} \in \mathbf{R}^{n \times n}, i=$ $0,1,2, \cdots, p$, the following statement is true: $\zeta^{\mathrm{T}} F_{0} \zeta>0$ for any $\zeta \in \mathbf{R}^{n}$ satisfying $\zeta^{\mathrm{T}} F_{i} \zeta \geqslant 0$, if there exist real scalars $\tau_{i} \geqslant 0, i=0,1,2, \cdots, p$, such that

$$
F_{0}-\sum_{i=1}^{p} \tau_{i} F_{i}>0
$$

For $p=1$, these two statements are equivalent.

\section{Main results}

In this section, we shall focus on the delay-dependent stability and passivity for system (1).

Theorem 1. For given scalars $\bar{d}, \gamma \geqslant 0$ and $0 \leqslant \mu \leqslant 1$, then, for any delay $d(t)$ satisfying (2), system (1) is stochastically stable and robustly passive, if there exist symmetric positive-definite matrices $Q, Z, \Lambda_{i}$, a scalar $\varepsilon_{i}$ and matrices $P_{i}, Y_{1 i}, Y_{2 i}, Y_{3 i}, Y_{4 i}, V_{1 i}, V_{2 i}$ and $V_{3 i}$ such that for each $i \in \mathbf{S}$,

$$
E^{\mathrm{T}} P_{i}=P_{i}^{\mathrm{T}} E \geqslant 0
$$

$\Theta_{i}=$

$$
\left[\begin{array}{cccccc}
\Xi_{i 11} & \Xi_{i 12} & \Xi_{i 13} & \bar{d} Y_{1 i} & \Xi_{i 15} & V_{1 i}^{\mathrm{T}} B_{i}-C_{z i}^{\mathrm{T}} \\
* & \Xi_{i 22} & \Xi_{i 23} & \bar{d} Y_{2 i} & V_{2 i}^{\mathrm{T}} D_{i}-V_{3 i} & V_{2 i}^{\mathrm{T}} B_{i} \\
* & * & \Xi_{i 33} & \bar{d} Y_{3 i} & \Xi_{i 35} & -C_{d z i}^{\mathrm{T}} \\
* & * & * & -\bar{d} Z & \bar{d} Y_{4 i}^{\mathrm{T}} & 0 \\
* & * & * & * & \Xi_{i 55} & \Lambda_{i} C_{i} B_{i}+V_{3 i}^{\mathrm{T}} B_{i} \\
* & * & * & * & * & -\gamma I-B_{z i}^{\mathrm{T}}-B_{z i}
\end{array}\right]<0
$$

where

$$
\begin{aligned}
& \Xi_{i 11}=\sum_{j=1}^{N} \pi_{i j} E^{\mathrm{T}} P_{j}+V_{1 i}^{\mathrm{T}} A_{i}+A_{i}^{\mathrm{T}} V_{1 i}+Y_{1 i} E+E^{\mathrm{T}} Y_{1 i}^{\mathrm{T}}+Q \\
& \Xi_{i 12}=P_{i}^{\mathrm{T}}-V_{1 i}^{\mathrm{T}}+A_{i}^{\mathrm{T}} V_{2 i}+E^{\mathrm{T}} Y_{2 i}^{\mathrm{T}} \\
& \Xi_{i 13}=-Y_{1 i} E+V_{1 i}^{\mathrm{T}} A_{d i}+E^{\mathrm{T}} Y_{3 i}^{\mathrm{T}} \\
& \Xi_{i 15}=A_{i}^{\mathrm{T}} C_{i}^{\mathrm{T}} \Lambda_{i}+E^{\mathrm{T}} Y_{4 i}^{\mathrm{T}}+V_{1 i}^{\mathrm{T}} D_{i}+A_{i}^{\mathrm{T}} V_{3 i}+\varepsilon_{i} C_{i}^{\mathrm{T}} K_{i}^{\mathrm{T}} \\
& \Xi_{i 22}=-V_{2 i}-V_{2 i}^{\mathrm{T}}+\bar{d} Z \\
& \Xi_{i 23}=-Y_{2 i} E+V_{2 i}^{\mathrm{T}} A_{d i} \\
& \Xi_{i 33}=-(1-\mu) Q-Y_{3 i} E-E^{\mathrm{T}} Y_{3 i}^{\mathrm{T}} \\
& \Xi_{i 35}=A_{d i}^{\mathrm{T}} C_{i}^{\mathrm{T}} \Lambda_{i}-E^{\mathrm{T}} Y_{4 i}^{\mathrm{T}}+A_{d i}^{\mathrm{T}} V_{3 i} \\
& \Xi_{i 55}=\Lambda_{i} C_{i} D_{i}+D_{i}^{\mathrm{T}} C_{i}^{\mathrm{T}} \Lambda_{i}+V_{3 i}^{\mathrm{T}} D_{i}+D_{i}^{\mathrm{T}} V_{3 i}-2 \varepsilon_{i} I .
\end{aligned}
$$

Proof. First, we shall show system (1) is regular and impulse free.

Define

$$
\begin{aligned}
& \bar{E}=\left[\begin{array}{ll}
E & 0 \\
0 & 0
\end{array}\right], \quad \bar{A}_{i}=\left[\begin{array}{cc}
0 & I \\
A_{i} & -I
\end{array}\right], \\
& \bar{A}_{d i}=\left[\begin{array}{cc}
0 & 0 \\
A_{d i} & 0
\end{array}\right], \quad \bar{P}_{i}=\left[\begin{array}{cc}
P_{i} & 0 \\
V_{1 i} & V_{2 i}
\end{array}\right] \\
& \bar{Y}_{1 i}=\left[\begin{array}{cc}
Y_{1 i} & 0 \\
Y_{2 i} & 0
\end{array}\right] \quad \bar{Y}_{3 i}=\left[\begin{array}{cc}
Y_{3 i} & 0 \\
0 & 0
\end{array}\right], \\
& \bar{Q}_{1}=\left[\begin{array}{cc}
Q & 0 \\
0 & \bar{d} Z
\end{array}\right], \quad \bar{Q}_{2}=\left[\begin{array}{cc}
(1-\mu) Q & 0 \\
0 & \bar{d} Z
\end{array}\right] .
\end{aligned}
$$

From (6), it is easy to see that

$$
\begin{gathered}
\bar{E}^{\mathrm{T}} \bar{P}_{i}=\bar{P}_{i}^{\mathrm{T}} \bar{E} \geqslant 0 \\
\pi_{i i} \bar{E}^{\mathrm{T}} \bar{P}_{i}+\bar{A}_{i}^{\mathrm{T}} \bar{P}_{i}+\bar{P}_{i}^{\mathrm{T}} \bar{A}_{i}+\bar{Y}_{1 i} \bar{E}+\bar{E}^{\mathrm{T}} \bar{Y}_{1 i}^{\mathrm{T}}<0 \\
{\left[\begin{array}{cc}
\bar{\Xi} & \bar{P}_{i}^{\mathrm{T}} \bar{A}_{d i}-\bar{Y}_{1 i} \bar{E}+\bar{E}^{\mathrm{T}} \bar{Y}_{3 i}^{\mathrm{T}} \\
* & -\bar{Q}_{2}-\bar{E}^{\mathrm{T}} \bar{Y}_{3 i}^{\mathrm{T}}-\bar{Y}_{3 i} \bar{E}
\end{array}\right]<0}
\end{gathered}
$$

where

$$
\bar{\Xi}=\pi_{i i} \bar{E}^{\mathrm{T}} \bar{P}_{i}+\bar{A}_{i}^{\mathrm{T}} \bar{P}_{i}+\bar{P}_{i}^{\mathrm{T}} \bar{A}_{i}+\bar{Q}_{1}+\bar{Y}_{1 i} \bar{E}+\bar{E}^{\mathrm{T}} \bar{Y}_{1 i}^{\mathrm{T}} .
$$

Since $\operatorname{rank}(E)=r \leqslant n$, there exist nonsingular matrices $G$ and $H$, such that $G E H=\left[\begin{array}{cc}I_{r} & 0 \\ 0 & 0\end{array}\right]$.

Denote

$$
\begin{gathered}
G \bar{A}_{i} H=\left[\begin{array}{cc}
A_{i 11} & A_{i 12} \\
A_{i 21} & A_{i 22}
\end{array}\right] \\
G^{-\mathrm{T}} \bar{P}_{i} H=\left[\begin{array}{ll}
P_{i 11} & P_{i 12} \\
P_{i 21} & P_{i 22}
\end{array}\right] \\
H^{\mathrm{T}} \bar{Y}_{1 i} G^{-1}=\left[\begin{array}{ll}
Y_{i 11} & Y_{i 12} \\
Y_{i 21} & Y_{i 22}
\end{array}\right]
\end{gathered}
$$

for every $i \in \mathbf{S}$. From (7a), we can deduce that $P_{i 22}=0$ for each $i \in \mathbf{S}$. Pre-multiplying and post-multiplying (7b) by $H^{\mathrm{T}}$ and $H$, respectively, we have

$$
A_{i 22}^{\mathrm{T}} P_{i 22}+P_{i 22}^{\mathrm{T}} A_{i 22}<0 .
$$


According to the proof of Theorem 1 in [7], we have from (8) that the pair $\left(E, A_{i}\right)$ is regular and impulse free for each $i \in \mathbf{S}$. Pre-multiplying and post-multiplying (7c) by $\left[\begin{array}{ll}I & I\end{array}\right]$ and $\left[\begin{array}{ll}I & I\end{array}\right]^{\mathrm{T}}$, respectively, we get

$$
\pi_{i i} \bar{E}^{\mathrm{T}} \bar{P}_{i}+\left(\bar{A}_{i}+\bar{A}_{d i}\right)^{\mathrm{T}} \bar{P}_{i}+\bar{P}_{i}^{\mathrm{T}}\left(\bar{A}_{i}+\bar{A}_{d i}\right)<0 .
$$

Using the above approach, from (7a) and (9), we can get that the pair $\left(E, A_{i}+A_{d i}\right)$ is regular and impulse free for each $i \in \mathbf{S}$. From Definition 1, system (1) with $u(t)=0$ is regular and impulse free for any delay $d(t)$ satisfying (2).

Next, we shall prove the stochastic stability of the system (1) with $w(t)=0$. Define a new process $\left\{\left(x_{t}, r_{t}\right), t \geqslant 0\right\}$ by $x_{t}(s)=x(t+s),-2 \bar{d} \leqslant s \leqslant 0$, then $\left\{\left(x_{t}, r_{t}\right), t \geqslant 0\right\}$ is a Markov process with initial state $\left(\phi(\cdot), r_{0}\right)$. Now, for $t \geqslant \bar{d}$, choose a stochastic Lyapunov functional candidate as

$$
\begin{aligned}
& V\left(x_{t}, r_{t}, t\right)=x^{\mathrm{T}}(t) E^{\mathrm{T}} P\left(r_{t}\right) E x(t)+ \\
& \int_{t-d(t)}^{t} x^{\mathrm{T}}(\alpha) Q x(\alpha) \mathrm{d} \alpha+ \\
& \int_{-\bar{d}}^{0} \int_{t+\beta}^{t} \dot{x}^{\mathrm{T}}(\alpha) E^{\mathrm{T}} Z E \dot{x}(\alpha) \mathrm{d} \alpha \mathrm{d} \beta+ \\
& 2 \sum_{j=1}^{m} \lambda_{i j} \int_{0}^{\sigma_{i j}} f_{i j}\left(\sigma_{i j}\right) \mathrm{d} \sigma_{i j} .
\end{aligned}
$$

Let $\boldsymbol{L}$ be the weak infinitesimal generator of the random $\operatorname{process}\left\{x_{t}, r_{t}\right\}$. Then, for each $i \in \mathbf{S}$, we have

$$
\begin{aligned}
& \boldsymbol{L} V\left(x_{t}, i, t\right) \leqslant 2 x^{\mathrm{T}}(t) E^{\mathrm{T}} P_{i} \dot{x}(t)+x^{\mathrm{T}}(t)\left(\sum_{j=1}^{N} \pi_{i j} E^{\mathrm{T}} P_{j}\right) x(t)+ \\
& x^{\mathrm{T}}(t) Q x(t)-(1-\mu) x^{\mathrm{T}}(t-d(t)) Q x(t-d(t))+ \\
& \bar{d} \dot{x}^{\mathrm{T}}(t) E^{\mathrm{T}} Z E \dot{x}(t)-\int_{t-d(t)}^{t} \dot{x}^{\mathrm{T}}(\alpha) E^{\mathrm{T}} Z E \dot{x}(\alpha) \mathrm{d} \alpha+ \\
& 2 f_{i}^{\mathrm{T}}\left(\sigma_{i}\right) \Lambda_{i} C_{i}\left[A_{i} x(t)+A_{d i}(t-d(t))+D_{i} f_{i}\left(\sigma_{i}\right)\right]+ \\
& 2\left[x^{\mathrm{T}}(t) Y_{1 i}+(E \dot{x}(t))^{\mathrm{T}} Y_{2 i}+x^{\mathrm{T}}(t-d(t)) Y_{3 i}+f_{i}^{\mathrm{T}}\left(\sigma_{i}\right) Y_{4 i}\right] \times \\
& {\left[E x(t)-E x(t-d(t))-\int_{t-d(t)}^{t} E \dot{x}(\alpha) \mathrm{d} \alpha\right]+} \\
& \quad\left[x^{\mathrm{T}}(t) V_{1 i}^{\mathrm{T}}+(E \dot{x}(t))^{\mathrm{T}} V_{2 i}^{\mathrm{T}}+f_{i}^{\mathrm{T}}\left(\sigma_{i}\right) V_{3 i}^{\mathrm{T}}\right] \times \\
& {\left[-E \dot{x}(t)+A_{i} x(t)+A_{d i}(t-d(t))+D_{i} f_{i}\left(\sigma_{i}\right)\right] .}
\end{aligned}
$$

It follows from Jensen integral inequality ${ }^{[19]}$ that

$$
-\int_{t-d(t)}^{t} \dot{x}^{\mathrm{T}}(\alpha) E^{\mathrm{T}} Z E \dot{x}(\alpha) \mathrm{d} \alpha \leqslant \zeta^{\mathrm{T}}(t)(-\bar{d} Z) \zeta(t)
$$

where $\zeta(t)=-\int_{t-d(t)}^{t} \frac{1}{d} E \dot{x}(\alpha) \mathrm{d} \alpha$.

From (11), we have that, for each $i \in \mathbf{S}$

$$
\boldsymbol{L} V\left(x_{t}, i, t\right) \leqslant \boldsymbol{X}^{\mathrm{T}} \Phi_{i 0} \boldsymbol{X}
$$

where

$$
\begin{aligned}
& \boldsymbol{X}=\left[x^{\mathrm{T}}(t) \dot{x}^{\mathrm{T}} E^{\mathrm{T}} x^{\mathrm{T}}(t-d(t)) \zeta^{\mathrm{T}}(t) f_{i}^{\mathrm{T}}\left(\sigma_{i}\right)\right]^{\mathrm{T}} \\
& \Phi_{i 0}=\left[\begin{array}{ccccc}
\Xi_{i 11} & \Xi_{i 12} & \Xi_{i 13} & \bar{d} Y_{1 i} & \Xi_{i 15}-\varepsilon_{i} C_{i}^{\mathrm{T}} K_{i}^{\mathrm{T}} \\
* & \Xi_{i 22} & \Xi_{i 23} & \bar{d} Y_{2 i} & V_{2 i}^{\mathrm{T}} D_{i}-V_{3 i} \\
* & * & \Xi_{i 33} & \bar{d} Y_{3 i} & A_{d i}^{\mathrm{T}} C_{i}^{\mathrm{T}} \Lambda_{i}-E^{\mathrm{T}} Y_{4 i}^{\mathrm{T}}+A_{d i}^{\mathrm{T}} V_{3 i} \\
* & * & * & -\bar{d} Z & \bar{d} Y_{4 i}^{\mathrm{T}} \\
* & * & * & * & \Xi_{i 55}+2 \varepsilon_{i} I
\end{array}\right] .
\end{aligned}
$$

In addition, from (3), we have

$$
f_{i}^{\mathrm{T}}\left(\sigma_{i}\right)\left[K_{i} C_{i} x(t)-f_{i}\left(\sigma_{i}\right)\right] \geqslant 0 .
$$

By applying S-procedure ${ }^{[18]}$, we can see that $\boldsymbol{L} V\left(x_{t}, i, t\right)<0$ for $\boldsymbol{X} \neq 0$ is implied by the existence of a scalar $\varepsilon_{i}$ for each $i \in \mathbf{S}$ such that

$$
\boldsymbol{X}^{\mathrm{T}} \Phi_{i 0} \boldsymbol{X}+2 \varepsilon_{i} f_{i}^{\mathrm{T}}\left(\sigma_{i}\right)\left[K_{i} C_{i} x(t)-f_{i}\left(\sigma_{i}\right)\right]<0, \quad \forall \boldsymbol{X} \neq \mathbf{0} .
$$

That is

$$
\boldsymbol{L} V\left(x_{t}, i, t\right) \leqslant \boldsymbol{X}^{\mathrm{T}} \Phi_{i 1} \boldsymbol{X}<\mathbf{0}, \quad \forall \boldsymbol{X} \neq \mathbf{0}
$$

where

$$
\Phi_{i 1}=\Phi_{i 0}+\left[\begin{array}{ccccc}
0 & 0 & 0 & 0 & \varepsilon_{i} C_{i}^{\mathrm{T}} K_{i}^{\mathrm{T}} \\
* & 0 & 0 & 0 & 0 \\
* & * & 0 & 0 & 0 \\
* & * & * & 0 & 0 \\
* & * & * & * & -2 \varepsilon I
\end{array}\right]
$$

From (14), there exists a small scalar $\rho>0$ such that for each $i \in \mathbf{S}, \boldsymbol{L} V\left(x_{t}, i, t\right) \leqslant-\rho\|x(t)\|^{2}$.

Therefore, for any $t \geqslant \bar{d}$, by Dynkin's formula, we obtain

$$
\boldsymbol{E} V\left(x_{t}, i, t\right)-\boldsymbol{E} V\left(x_{\bar{d}}, r_{\bar{d}}, \bar{d}\right) \leqslant-\rho \boldsymbol{E} \int_{\bar{d}}^{t}\|x(s)\|^{2} \mathrm{~d} s
$$

which yields

$$
\boldsymbol{E} \int_{\bar{d}}^{t}\|x(s)\|^{2} \mathrm{~d} s \leqslant \rho^{-1} \boldsymbol{E} V\left(x_{\bar{d}}, r_{\bar{d}}, \bar{d}\right) .
$$

From [18], the regularity and the absence of impulses of pair $\left(E, A_{i}\right)$ for each $i \in \mathbf{S}$ implies there exist two invertible matrices $M_{i}$ and $N_{i}$ such that

$$
\begin{aligned}
& M_{i} E N_{i}=\left[\begin{array}{ll}
I_{r} & 0 \\
0 & 0
\end{array}\right] \\
& M_{i} A_{i} N_{i}=\left[\begin{array}{cc}
A_{i 1} & 0 \\
0 & I_{n-r}
\end{array}\right] \\
& M_{i} A_{d i} N_{i}=\left[\begin{array}{ll}
A_{i d 1} & A_{i d 2} \\
A_{i d 3} & A_{i d 4}
\end{array}\right] \\
& M_{i} D_{i}=\left[\begin{array}{c}
D_{i 1} \\
D_{i 2}
\end{array}\right] \\
& \bar{C}_{i}=C_{i} N_{i}^{-1} .
\end{aligned}
$$

Then, for each $i \in \mathbf{S}$, system (1) is decomposed as

$$
\begin{aligned}
\dot{\xi}_{1}(t)= & A_{i 1} \xi_{1}(t)+A_{i d 1} \xi_{1}(t-d(t))+ \\
& A_{i d 2} \xi_{2}(t-d(t))+D_{i 1} f_{i}\left(\eta_{i}\right) \\
-\xi_{2}(t)= & A_{i d 3} \xi_{1}(t-d(t))+A_{i d 4} \xi_{2}(t-d(t))+D_{i 2} f_{i}\left(\eta_{i}\right) \\
\xi(t)= & \psi(t)=N_{i}^{-1} \phi(t), t \in[-\bar{d}, 0]
\end{aligned}
$$

where $\eta_{i}=\bar{C}_{i} \xi(t), \xi(t)=\left[\begin{array}{l}\xi_{1}(t) \\ \xi_{2}(t)\end{array}\right]=N_{i}^{-1} x(t)$.

Expression (3) can be equivalently described as

$\sigma_{i j} f_{i j}\left(\sigma_{i j}\right)\left(\sigma_{i j} f_{i j}\left(\sigma_{i j}\right)-k_{j} \sigma_{i j}^{2}\right) \leqslant 0(j=1,2, \cdots, m) \Rightarrow$

$f_{i j}^{2}\left(\sigma_{i j}\right) \leqslant k_{j}^{2} \sigma_{i j}^{2} \Rightarrow\left\|f_{i}\left(\sigma_{i}\right)\right\|^{2} \leqslant\left\|K_{i} C_{i} x(t)\right\|^{2}$. 
Therefore,

$$
f_{i}^{\mathrm{T}} f_{i} \leqslant \xi^{\mathrm{T}}(t) \bar{C}_{i}^{\mathrm{T}} K_{i}^{\mathrm{T}} K_{i} \bar{C}_{i} \xi(t) .
$$

From (17), we can deduce that

$$
\begin{aligned}
\left\|D_{i 1} f_{i}\left(\eta_{i}\right)\right\|^{2} \leqslant & \left\|D_{i 1} K_{i} \bar{C}_{i} \xi(t)\right\|^{2} \Rightarrow \\
& \left\|D_{i 1} f_{i}\left(\eta_{i}\right)\right\| \leqslant \sqrt{k_{1}}\left(\xi_{1}(t)+\xi_{2}(t)\right) \\
\left\|D_{i 1} f_{i}\left(\eta_{i}\right)\right\|^{2} \leqslant & \left\|D_{i 2} K_{i} \bar{C}_{i} \xi(t)\right\|^{2} \Rightarrow \\
& \left\|D_{i 2} f_{i}\left(\eta_{i}\right)\right\| \leqslant \sqrt{k_{2}}\left(\xi_{1}(t)+\xi_{2}(t)\right) .
\end{aligned}
$$

where

$$
\begin{aligned}
& k_{1}=\lambda_{\max , i \in \mathbf{S}}\left[\bar{C}_{i}^{\mathrm{T}} K_{i}^{\mathrm{T}} D_{i 1}^{\mathrm{T}} D_{i 1} K_{i} \bar{C}_{i}\right] \geqslant 0 \\
& k_{2}=\lambda_{\max , i \in \mathbf{S}}\left[\bar{C}_{i}^{\mathrm{T}} K_{i}^{\mathrm{T}} D_{i 2}^{\mathrm{T}} D_{i 2} K_{i} \bar{C}_{i}\right] \geqslant 0 .
\end{aligned}
$$

For any $0 \leqslant t \leqslant \bar{d}$, it follows from (16) that

$$
\begin{gathered}
\left\|\xi_{1}(t)\right\| \leqslant\left\|\xi_{1}(0)\right\|+k_{3} \int_{0}^{t}\left[\left\|\xi_{1}(s)\right\|+\left\|\xi_{1}(s-d(t))\right\|+\right. \\
\left.\left\|\xi_{2}(s)\right\|+\left\|\xi_{2}(s-d(t))\right\|\right] \mathrm{d} s \leqslant \\
\left(2 k_{3} \bar{d}+1\right)\|\psi\|_{\bar{d}}+k_{3} \int_{0}^{t}\left\|\xi_{1}(s)\right\| \mathrm{d} s
\end{gathered}
$$

where $k_{3}=\max _{i \in \mathbf{S}}\left\{\left\|A_{i 1}\right\|+\sqrt{k_{1}},\left\|A_{i d 1}\right\|,\left\|A_{i d 2}\right\|\right\} \geqslant 0$.

By applying the Gronwall-Bellman Lemma, we obtain from (18) that for any $0 \leqslant t \leqslant \bar{d}$,

$$
\sup _{0 \leqslant t \leqslant \bar{d}}\left\|\xi_{1}(t)\right\| \leqslant\left(2 k_{3} \bar{d}+1\right)\|\psi\|_{\bar{d}} \mathrm{e}^{k_{3} \bar{d}} .
$$

Consider (16) and (19), it can be deduced that $\sup _{0 \leqslant t \leqslant \bar{d}}\left\|\xi_{2}(t)\right\|$ is bounded. We assume $0 \leqslant \sqrt{k_{2}}<1$, then for any $0 \leqslant t \leqslant \bar{d}$, we have

$$
\sup _{0 \leqslant t \leqslant \bar{d}}\left\|\xi_{2}(t)\right\|^{2} \leqslant\left(1-\sqrt{k_{2}}\right)^{-2} k_{4}^{2}\left(\left(2 k_{3} \bar{d}+1\right) e^{k_{3} \bar{d}}+2\right)^{2}\|\psi\|_{\bar{d}}
$$

where $k_{4}=\max _{i \in \mathbf{S}}\left\{\left\|A_{i d 3}\right\|,\left\|A_{i d 4}\right\|, \sqrt{k_{2}}\right\}$. Hence,

$$
\sup _{0 \leqslant t \leqslant \bar{d}}\|\xi(t)\|^{2} \leqslant \sup _{0 \leqslant t \leqslant \bar{d}}\left\|\xi_{1}(t)\right\|^{2}+\sup _{0 \leqslant t \leqslant \bar{d}}\left\|\xi_{2}(t)\right\|^{2} \leqslant k_{5}\|\psi\|_{\bar{d}}^{2}
$$

where

$$
\begin{aligned}
k_{5}= & \left(2 k_{3} \bar{d}+1\right)^{2} \mathrm{e}^{2 k_{3} \bar{d}}+\left(1-\sqrt{k_{2}}\right)^{-2} k_{4}^{2} \times \\
& \left(\left(2 k_{3} \bar{d}+1\right) \mathrm{e}^{k_{3} \bar{d}}+2\right)^{2} .
\end{aligned}
$$

Therefore,

$$
\sup _{0 \leqslant t \leqslant \bar{d}}\|x(t)\|^{2} \leqslant k_{5}\left\|N_{i}\right\|^{2}\left\|N_{i}^{-1}\right\|^{2}\|\phi\|_{\bar{d}}^{2} .
$$

Expressions (15) and (20) imply the existence of a scalar $k_{6} \geqslant 0$ such that

$$
\begin{aligned}
& \boldsymbol{E} \int_{0}^{t}\|x(s)\|^{2} \mathrm{~d} s= \\
& \quad \boldsymbol{E} \int_{0}^{\bar{d}}\|x(s)\|^{2} \mathrm{~d} s+\boldsymbol{E} \int_{\bar{d}}^{t}\|x(s)\|^{2} \mathrm{~d} s \leqslant k_{6} \boldsymbol{E}\|\phi\|_{\bar{d}}^{2} .
\end{aligned}
$$

From Definition 1, we get that system (1) with $w(t)=0$ is stochastically stable.
Now we shall show the passivity property of system (1) for each $i \in \mathbf{S}$.

Under zero initial condition, it is easy to see that

$$
\begin{aligned}
& \boldsymbol{L} V\left(x_{t}, i, t\right)-2 w^{\mathrm{T}}(t) z(t)-\gamma w^{\mathrm{T}}(t) w(t)= \\
& \boldsymbol{L} V\left(x_{t}, i, t\right)-\gamma w^{\mathrm{T}}(t) w(t)- \\
& 2 w^{\mathrm{T}}(t)\left[C_{z}\left(r_{t}\right) x(t)+C_{d z}\left(r_{t}\right) x(t-d(t))+B_{z}\left(r_{t}\right) w(t)\right] \leqslant \\
& \overline{\boldsymbol{X}}^{\mathrm{T}}(s) \Theta_{i} \overline{\boldsymbol{X}}(s)
\end{aligned}
$$

where

$$
\overline{\boldsymbol{X}}=\left[x^{\mathrm{T}}(t) \dot{x}^{\mathrm{T}} E^{\mathrm{T}} x^{\mathrm{T}}(t-d(t)) \zeta^{\mathrm{T}}(t) f_{i}^{\mathrm{T}}\left(\sigma_{i}\right) w^{\mathrm{T}}(t)\right]^{\mathrm{T}} .
$$

From (6b), we get

$$
\boldsymbol{L} V\left(x_{t}, i, t\right)-2 w^{\mathrm{T}}(t) z(t)-\gamma w^{\mathrm{T}}(t) w(t)<0 .
$$

Integrating both sides of $(21)$ with respect to $t$ over the time period $\left[0, t^{*}\right]$ gives rise to

$$
\begin{gathered}
V\left(x_{t^{*}}, i, t^{*}\right)-V\left(x_{0}, i, 0\right)-\boldsymbol{E}\left\{2 \int_{0}^{t^{*}} w^{\mathrm{T}}(s) z(s) \mathrm{d} s\right\}- \\
\gamma \int_{0}^{t^{*}} w^{\mathrm{T}}(s) w(s) \mathrm{d} s \leqslant 0 .
\end{gathered}
$$

Then, from Definition 2, system (1) is robustly passive.

Remark 1. Theorem 1 gives a delay-dependent sufficient condition of the stochastic stability with passivity for Lur'e singular system with Markovian switching (1). In the derivation of the delay-dependent result in Theorem 1 , no model transformation is performed onto system (1). Moreover, we have introduced some free-weighting matrices, (this approach is also known as the slack matrix approach), which may help to reduce conservatism.

\section{Numerical examples}

In this section, two numerical examples are presented to illustrate the effectiveness of the proposed method.

Example 1. Consider system (1) with one mode and the system parameters are described as

$$
\begin{aligned}
& A_{1}=\left[\begin{array}{cr}
-0.5 & 0 \\
0 & -1
\end{array}\right], \quad A_{d 1}=\left[\begin{array}{cc}
-1.1 & 1 \\
0 & 0.5
\end{array}\right], \\
& D_{1}=\left[\begin{array}{l}
0.2 \\
0.1
\end{array}\right], \quad B_{1}=\left[\begin{array}{l}
0.1 \\
0.2
\end{array}\right], \\
& C_{1}=\left[\begin{array}{ll}
0.6 & 0.8
\end{array}\right], \quad C_{d z 1}=\left[\begin{array}{ll}
0.6 & 0.8
\end{array}\right], \\
& C_{d z 1}=\left[\begin{array}{ll}
0.1 & 0.1
\end{array}\right], \quad B_{z 1}=0.3 \\
& K_{1}=0.4
\end{aligned}
$$

When $w(t)$ is not considered, the maximum allowable delay bound $\bar{d}$ for different $\mu$ is shown in Table 1 . For comparison, the upper bounds obtained for the criteria in [9] are also listed in Table 1. It can be seen that our method is less conservative than those in [9].

Table 1. Maximum allowable time delay $\bar{d}$ for different $\mu$

\begin{tabular}{cccccc}
\hline$\mu$ & 0 & 0.3 & 0.6 & 0.7 & 1.0 \\
\hline $\bar{d}([9])$ & 1.07 & 1.02 & 0.93 & 0.86 & 0.80 \\
$\bar{d}$ (This paper) & 2.09 & 1.71 & 1.36 & 1.24 & 0.91 \\
\hline
\end{tabular}


Taking disturbance $w(t)$ into account, Table 2 provides the maximum allowed time-delay $\bar{d}$ for different $\gamma \geqslant 0$ when $\mu=0.5$ according to Theorem 1 .

Table 2. Maximum allowable time delay $\bar{d}$ for different $\gamma$

\begin{tabular}{ccccccc}
\hline$\gamma$ & 0 & 0.2 & 0.5 & 0.8 & 1 & 1.2 \\
\hline $\bar{d}$ & 4.12 & 3.92 & 2.86 & 2.85 & 1.74 & 1.18 \\
\hline
\end{tabular}

Example 2. Consider the system (1) with two modes, i.e., $N=2$, the system parameters are described as

$$
\begin{aligned}
& A_{1}=\left[\begin{array}{cc}
-0.3 & 0.1 \\
0.1 & -1
\end{array}\right], \quad A_{2}=\left[\begin{array}{cc}
0.4 & 0.2 \\
0 & -0.9
\end{array}\right], \\
& A_{d 1}=\left[\begin{array}{ll}
-1.4 & 0.5 \\
0.1 & 0.7
\end{array}\right], \quad A_{d 2}=\left[\begin{array}{ll}
-1.3 & 0.6 \\
0.0 & 0.9
\end{array}\right], \\
& B_{1}=\left[\begin{array}{l}
0.01 \\
0.02
\end{array}\right], \quad B_{2}=\left[\begin{array}{l}
0.01 \\
0.02
\end{array}\right], \\
& D_{1}=D_{2}=0 .
\end{aligned}
$$

The rate matrix $\Pi$ and singular matrix $E$ is chosen as follows:

$$
\begin{aligned}
& \Pi=\left[\begin{array}{cc}
-0.5 & 0.5 \\
0.3 & -0.3
\end{array}\right] \\
& E=\left[\begin{array}{ll}
1 & 0 \\
0 & 0
\end{array}\right] .
\end{aligned}
$$

The comparison of maximum time delay upper bound $\bar{d}$ via different method is recorded in Table 3 .

Table 3. Maximum allowable time delay $\bar{d}$ for different $\mu$

\begin{tabular}{ccccc}
\hline$\mu$ & 0 & 0.4 & 0.7 & 1 \\
\hline $\bar{d}([13])$ & 1.34 & 0.79 & 0.27 & Infeasible \\
$\bar{d}$ (This paper) & 1.95 & 1.23 & 0.86 & 0.31 \\
\hline
\end{tabular}

It can be seen from Table 3 that our results have less conservatism than the recent results for time-delay singular Markovian jump systems in [13].

\section{Conclusions}

The problem of stochastic stability and passivity for a class of Lur'e singular systems with time-varying delay and Markovian switching has been investigated. The delaydependent condition has been derived in terms of LMI, in which some free-weighting matrices have been employed to express the relationship between the terms in the LeibnizNewton formula. Finally, numerical examples have been presented to show the effectiveness and superiority of the proposed method.

\section{References}

[1] Y. He, M. Wu, J. H. She, G. P. Liu. Robust stability for delay Lure control systems with multiple nonlinearities. Journal of Computational and Applied Mathematics, vol. 176, no. 2, pp. 371-380, 2005.
[2] Q. L. Han. A new delay-dependent absolute stability criterion for a class of nonlinear neutral systems. Automatica, vol. 44, no. 1, pp. 272-277, 2008.

[3] Q. L. Han, A. K. Xue, S. R. Liu, X. H. Yu. Robust absolute stability criteria for uncertain Lur'e systems of neutral type. International Journal of Robust and Nonlinear Control, vol. 18, no. 3, pp. 278-295, 2008.

[4] R. Q. Lu, H. Y. Su, J. Chu. Robust $H_{\infty}$ control for a class of uncertain Lur'e singular systems with time-delays. In Proceedings of the 42nd IEEE Conference on Decision and Control, IEEE, Hawaii, USA, pp. 5585-5590, 2003.

[5] Q. S. Meng, A. K. Xue, D. Y. Li, R. Q. Lu. Robust stability and stabilization for a class of uncertain Lur'e singular delay systems based on non-direction robot. In Proceedings of the 2006 IEEE International Conference on Control Applications, IEEE, Munich, Germany, pp. 3223-3228, 2006.

[6] R. Q. Lu, X. Z. Dai, H. Y. Su, J. Chu, A. K. Xue. Delaydependent robust stability and stabilization conditions for a class of Lur'e singular time-delay systems. Asian Journal of Control, vol. 10, no. 4, pp. 462-469, 2008.

[7] H. J. Wang, A. K. Xue, R. Q. Lu. Absolute stability criteria for a class of nonlinear singular systems with time delay. Nonlinear Analysis: Theory, Methods \& Applications, vol. 70, no. 2, pp. 621-630, 2009.

[8] C. Y. Yang, J. Xin, Q. L. Zhang, L. N. Zhou. Practical stability analysis and synthesis of linear descriptor systems with disturbances. International Journal of Automation and Computing, vol. 5, no. 2, pp. 138-144, 2008.

[9] H. J. Wang, A. K. Xue. Delay-dependent absolute stability criteria for uncertain Lur'e singular systems with timevarying delay. In Proceedings of 2009 American Control Conference, IEEE, Piscataway, NJ, USA, pp.4079-4084, 2009.

[10] H. J. Wang, A. K. Xue. Absolute stability criteria for Lur'e singular systems of neutral type. In Proceedings of the 29th Chinese Control Conference, IEEE, Beijing, China, pp. 1879-1884, 2010.

[11] Y. Q. Xia, E. K. Boukas, P. Shi, J. H. Zhang. Stability and stabilization of continuous-time singular hybrid systems. Automatica, vol. 45, no. 6, pp. 1504-1509, 2009.

[12] L. G. Wu, P. Shi, H. J. Gao. State estimation and slidingmode control of Markovian jump singular systems. IEEE Transactions on Automatic Control, vol. 55, no. 5, pp. 1213$1219,2010$.

[13] Z. G. Wu, H. Y. Su, J. Chu. Delay-dependent $H_{\infty}$ filtering for singular Markovian jump time-delay systems. Signal Processing, vol. 90, no. 6, pp. 1815-1824, 2010.

[14] X. D. Zhao, Q. S. Zeng. Delay-dependent stability analysis for Markovian jump systems with interval time-varyingdelays. International Journal of Automation and Computing, vol. 7, no. 2, pp. 224-229, 2010. 
[15] X. D. Zhao, Q. S. Zeng. $H_{\infty}$ output feedback control for stochastic systems with mode-dependent time-varying delays and Markovian jump parameters. International Journal of Automation and Computing, vol.7, no. 4, pp. 447-454, 2010.

[16] B. T. Cui, M. G. Hua. Observer-based passive control of linear time-delay systems with parametric uncertainty. Chaos, Solitons \& Fractals, vol. 32, no. 1, pp. 160-167, 2007.

[17] L. P. Liu, Z. Z. Han, W. L. Li. Non-fragile observer-based passive control for uncertain time delay systems subjected to input nonlinearity. Nonlinear Analysis: Theory, Methods \& Applications, vol. 73, no. 8, pp. 2603-2610, 2010.

[18] S. Boyd, L. El Ghaoui. Linear Matrix Inequalities in System and Control Theory, Philadelphia: SIAM, 1994.

[19] K. Q. Gu, V. L. Kharitonov, J. Chen. Stability of Timedelay Systems, Boston, Massachusetts, USA: Birkhäuser, 2003.

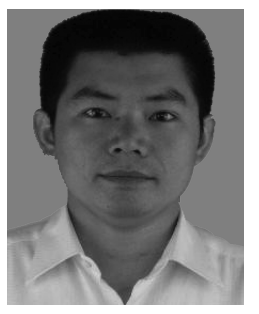

Ping-Fang Zhou received his B.Sc. degree in automation from Zhengzhou University of Technology, China in 1999. He received his M.Sc. degree in automation from Central South University, China in 2002. He received his Ph.D. degree in automation from Shanghai Jiao Tong University, China

in 2006 .

$\mathrm{He}$ is an associate professor in the School of Aeronautics and Astronautics in Shanghai Jiao Tong University, China.

His research interests include robust control, nonlinear control, real-time control, and flight control system.

E-mail: zhoupf@sjtu.edu.cn (Corresponding author)

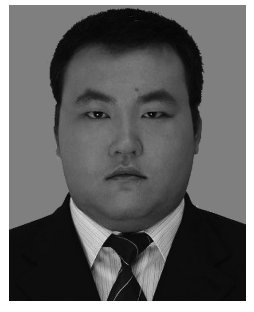

Yue-Ying Wang received the B.Sc. degree in mechanical engineering and automation from Beijing Institute of Technology, China in 2006 and the M. Sc. degree in navigation, guidance and control from Shanghai Jiao Tong University, China in 2010. He is now a Ph. D. candidate in instrument science and technology at Shanghai Jiao Tong University, China.

His research interests include robust control, filtering, and flight control system.

E-mail: wangyueyingsjtu@sjtu.edu.cn

Quan-Bao Wang received his $\mathrm{Ph}$. D. degree from Shanghai Jiao Tong University, Shanghai, China in 2010. He is an associate professor in the School of Aeronautics and Astronautics in Shanghai Jiao Tong University.

His research interests include measurement and control.

E-mail: quanbaowang@sjtu.edu.cn

Ji-An Chen received his $\mathrm{Ph}$. D. degree from Harbin Institute of Technology, Harbin, China in 1998. He is a professor at the School of Aeronautics and Astronautics in Shanghai Jiao Tong University.

His research interests include aircraft design, advanced materials and structures, and measurement.

E-mail: ja-chen@sjtu.edu.cn

Deng-Ping Duan received his Ph. D. degree from Harbin Institute of Technology, Harbin, China in 1996. He is a professor at the School of Aeronautics and Astronautics in Shanghai Jiao Tong University.

His research interests include aircraft design and integrated control.

E-mail: ddp@sjtu.edu.cn 